\title{
Parking Assist using Convolution Neural Networks
}

\author{
Swasthi B S, Anagha R, Arpitha S, Sanjay B S, Harshitha K
}

\begin{abstract}
Parking vehicles are one of the most frustrating tasks that people face these days. Locating an available parking space is a huge headache especially in urban areas. This paper aims to design one such parking system which, in many ways reduces the hassles of parking. The paper presents a system where a Machine Learning model, Convolution Neural Network(CNN) is used to classify parking slots in a parking space into vacant and filled slots. In order to optimize the task of classification, the method of Transfer Learning is implemented in the paper. The problem of parking stands not only limited to causing inconvenience to the drivers, but also escalates to much larger and extensive problems, affecting a lot more people the environment. Hence it is very important to have a system is used parking system in place. The model proposed in the paper sends across parking information to a driver well in advance, there by greatly reducing the waiting time for the vehicle.
\end{abstract}

Keywords: Convolution Neural Network (CNN),Reset, Transfer Learning, Feature extraction, Parking slot.

\section{INTRODUCTION}

All over the world, every year, the number of vehicles keeps increasing, but parking space for those vehicles remains constant. The hassles of parking here mainly pertain to carparking. The main reasons as to why parking is a cumbersome task is because of either lack of availability of parking space or lack of planning and discipline or both. Firstly, as number of vehicles increase, they need more space to park. But parking space is always a limited resource and cannot be expanded[1].Secondly, lack of discipline among drivers who park their car haphazardly, taking up more space than their vehicle needs, would further reduce the available parking space Needs, would further reduce the available parking space[2], and because of these reasons, looking for a vacant parking lot is a tiresome task for drivers. The consequences of this problem are even more damaging. As vehicles queue up waiting and looking for a vacant spot, the waiting vehicles cause traffic congestions, and traffic jams would further lead to wastage of fuel, time and effort [3]. Not just that, traffic jams would also risk increasing air pollution and noise pollution. The existing parking systems in most parking spaces usually involve a security person guiding the vehicles into vacant spots. But this system is quite time- consuming and complicated. Also it does not help in reducing the waiting time for vehicles.

Revised Manuscript Received on July 28, 2020.

* Correspondence Author

Swasthi B S*, Department of Computer Science and Engineering, Vidyavardhaka College of Engineering, Mysuru, India.

Anagha R, Department of Computer Science and Engineering, Vidyavardhaka College of Engineering, Mysuru, India.

Arpitha S, Department of Computer Science and Engineering, Vidyavardhaka College of Engineering, Mysuru, India.

Sanjay B S, Department of Computer Science and Engineering, Vidyavardhaka College of Engineering, Mysuru, India.

Harshitha K, Department of Computer Science and Engineering, Vidyavardhaka College of Engineering, Mysuru, India.

(C) The Authors. Published by Blue Eyes Intelligence Engineering and Sciences Publication (BEIESP). This is an open access article under the CC BY-NC-ND license (http://creativecommons.org/licenses/by-nc-nd/4.0/)
Researchers around the world have come up with IoT based systems, but they are difficult to implement and security of such systems would be an issue. The idea this paper presents looks to overcome the problems associated with parking [4]. This paper proposes a smart parking system that can be mainly beneficial to shopping complexes, school/college parking spaces. The model implements an image classifier using CNN to identify and classify parking spots into vacant and filled slots. This parking information is put across to driver's en-route, even before they reach the parking space, through a web application. The image classifier makes use of a pre-trained CNN architecture Alex Net. AlexNet-50 is a type of Convolution Neural Network which is made up of 50 layers. Also the application of Transfer Learning would enhance the efficiency of the CNN image classifier, thereby guaranteeing more accurate results. CNN is a Deep Learning algorithm which is predominantly used to process images and visual data[5].CNN is a type of Deep Neural Networks works by the principle of all putting weights to the properties of an image and learn to categorize the images based on the features, thus making it a very appropriate algorithm for classification tasks. Like many other neura networks have, CNN also comprises of multiple layers. They are input layer, output layer and between these two layers lay multiple hidden layers [6]. Since, CNNs generally have many layers of processing the efficiency of this particular model is higher than other algorithms. In addition to this, we have also used the Transfer Learning technology. Transfer learning is a machine learning technique where a model is fed on a dataset and trained.This learning gained by the model is reused and applied to a similar model. This technique basically helps a model keep track of everything it has learnt so far and use it in a similar scenario. This project models a parking spot detection system using a convolution neural network classifier running on the images of the parking space, and classifies those images as vacant and filled slots thereby helping the drivers to locate the most convenient and available parking slot in advance . This would greatly reduce the searching or waiting time for vehicles, implying that it also reduces the traffic jams at parking spaces. [7]

\section{METHODOLOGY}

\section{A. Existing system}

Today, in most parking spaces, the parking systems are mainly manual, i.e., the vehicles are guided to parking lots manually. In most shopping complexes and such places, vehicles once they enter the parking space have to wait in a long queue behind other vehicles, awaiting the issue of a receipt by a guard.

Published By:

Blue Eyes Intelligence Engineering

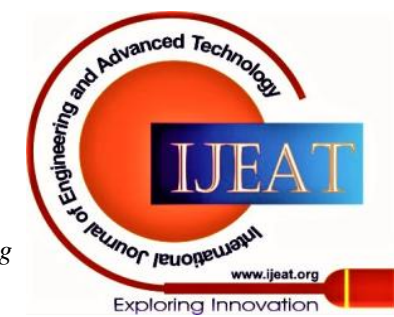


This is why traffic jams occur at parking spaces and may lead to increasing pollution. Once a vehicle clears the queue, the vehicle is lead to a vacant parking lot by another person. Although this looks to be a pretty convenient system, on the hind sight it is not. First, vehicles need to spend a lot of time waiting in the queue, and this would be very frustrating if it's a peak hour. Secondly, the rear chances that they might not actually find a vacant space even after entering the parking space. Also, since it is manual, the assisting person in the parking space can make mistakes in guiding vehicles to vacant slots. Any system which includes human intervention cannot prove to a hundred percent accurate. So, in reality the existing parking system is not the most effective system [8].

Researchers around the world have seen through the problems in the existing parking system, and have come up with other ideas and technologies to implement. One of the most common technologies looked into is Internet of Things (IoT), and IoT based parking systems are in action

in few places around the world. Although it might look to be a better option, this technology can also be flawed. First of all, any parking system developed using the IoT is very difficult to implement since it is a very complex technology to develop and implement [9]. Second, since IoT functions fully over the internet, security of the system is a major system, since the internet is no more a place, and the system would be prone to security breaches. Also, the cost of implementing an IoT based parking system would be pretty high. So these are some of the existing parking systems and the respective loopholes in them. Considering all the above problems and parameters, this project implements a much better and effective idea [10].

The parking system proposed in this paper is basically operated through a web application available to the users. The data required for the system is images of parking slots, comprising of bothvacantandfilledslots.About5000such parking images were considered as the training data. These images were fed to the Resnet-50 model where the process of Feature extraction would take place. Feature extraction is a process where the input data's dimensions are reduced such that the processing of the image becomes a lot easier that is, this process would extract only the required features or variables that are needed for the model[11]. So, ResNet here is mainly used to do Feature extraction, which is a very important aspect of the proposed model. Although Convolution Neural Networks are one of the most rewarding models, the main problem with it is that it has a lot of layers and when so many layers start converging, a degradation problem arises. As the network gets deeper and deeper, the accuracy reaches a peak and then begins to take a hit, which is appreciable. This condition is called the vanishing gradient problem. The ResNet-50 is a great solution to this problem. Residual neural networks avoid this problem by the technique of skip connections, or shortcuts. This technique is used to skip a few layers initially thereby reducing the total number of convolution layers. This technique also makes the learning process faster since it requires lesser time to propagate through lesser number of layers. This technique comes very handy when training very deep neural networks with complex data. This was the main reason behind choosing this Neural Network for the proposed system [12].

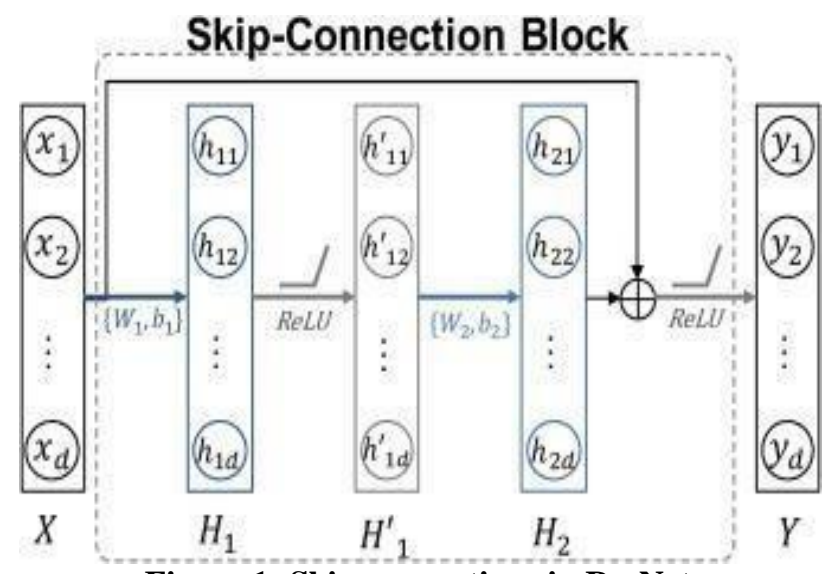

Figure 1: Skip connections in ResNet

Of the 5000 images considered for training, only a subset of these images was uploaded to the database due to the accommodation purpose of the database. These images in the database act as available parking slots in an area for the end users. So whenever the user wanted to know all possible parking slots close to his/her location, the images in the database would act as all those possible parking slots. During the Feature extraction process, we used the technique of Transfer learning to enhance the performance of the ResNet. This method helps the ResNet to use the knowledge learnt in one layer and apply it to the next layer, thereby improving the baseline performance. After feature extraction the classification of parking images into empty and filled slot images is done by the Decision Tree algorithm. Both these algorithms are most suitable for classification tasks, and since the number of classes involved here is two, it is not a very complex task for the algorithm.

\section{B. System Architecture}

The proposed system architecture consists of four modules each interconnected. They are Web Application, Database, Map API and CNN classifier. The user interacts with each of these modules either directly or indirectly.

Web Application: This module interacts directly with the user, through the user interface. The user interface provides the login, register features for the users and they have to let the application to fetch their current location. The web application has the Google Maps API integrated with it, in order to access current location of user. This web application has been built by using the Django framework for backend and suitable framework for frontend in the development of Full stack web application. The application shows all nearby vacant parking slots and also the nearest one among them.

Map API: The Project is integrated with the other modules of Web Application through the Google Map Application Programming Interface. We have utilized three Map APIs, the Google Maps Java script API which is used to fetch user's location, create a custom Is able map and the Distance Matrix API which is basically used to find the nearest vacant parking slots by computing

Published By:

Blue Eyes Intelligence Engineering

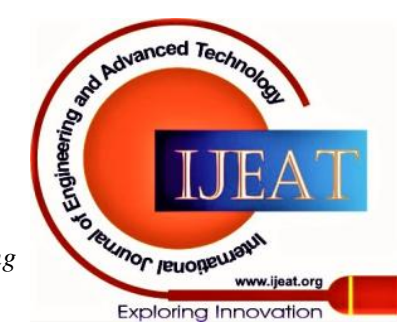


distance joining current user location and parking slots.

Third API used is the Directions API which creates a guiding route to the nearest slot.

Database: The database used in this project is the SQLite an disintegrated with the Django framework. This database basically has all the parking slots of a place and its location and slot status of each slot. The slot status of a parking spot is updated here in the database once the user accesses it. The Classifier is the one which actually evaluates the slot status and sends the same to the database. Apart from storing information about parking slots, the database also stores user authentication information.

Classifier: The Classifier module does the job of classifying the image into vacant or filled parking slots. The classifier uses both Decision Tree and Support Vector algorithms to classify parking images as empty or filled.

The Classifier model is directly operates in co-ordination with the MAP API module. The classifier takes the user location as input, searches for parking slot images in the database near user's location, classifies them and sends the empty parking slot status to the customer through the web app.

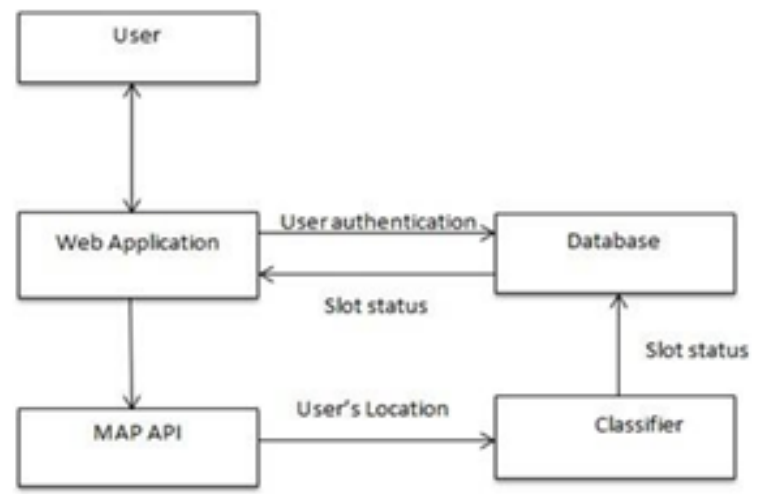

Figure 2: System Architecture

\section{Flow Diagram}

Figure 3 pictorially represents the operational flow of the proposed system through a flow chart .It represents the entire process of delivering parking information to users through the web applications well as the background running tasks including database access along with classification of the parking images. A few images comprising of both vacant and filled lot images are chosen at random from the dataset. These images undergo feature extraction through the ResNet-50 model and are then classified into the two classes.Infigure3,Parking Detection represents this whole process. These classified images are inserted into the database along with their location coordinates and class.

While all this happens at the background, the actual working of the system begins with the user accessing the web application by logging into the application. New users login after getting registered to application.

The user authentication details fetched from the database confirms the user login, if the login credentials are valid. The logged in user needs to permit the application to access his/her geographical location. If done so, then based on the user's location co-ordinates, empty parking slot images stored in the database is fetched and displayed over to the user. The user can see all empty parking slots nearby and also the near stone amongst them. The application displays only the empty parking slots nearby.

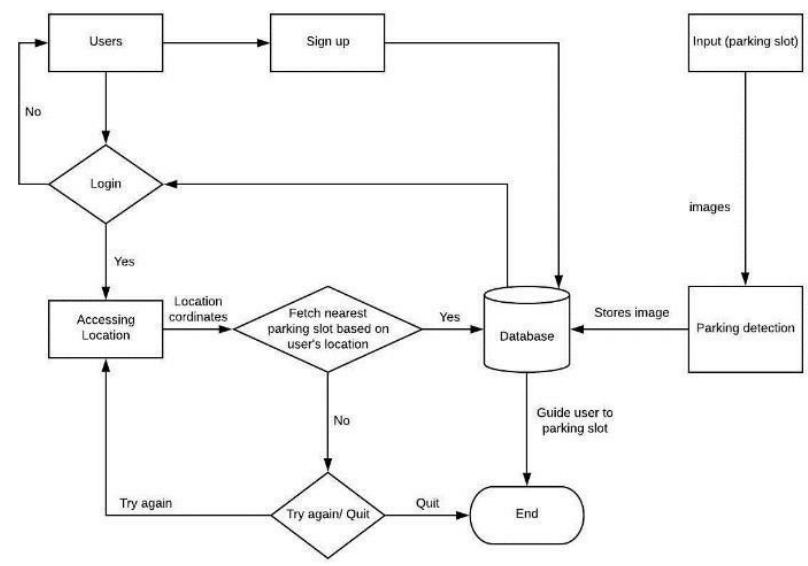

Fig 3: Flow Diagram

\section{RESULTS AND ANALYSIS}

The proposed system was implemented by simulating the system virtually. For the purpose of classification, other algorithms like Support Vector machine and Linear Regression were tried out along with Decision Tree algorithm. But the best accuracy was got by applying the Decision Tree algorithm. Also relative to other such models, decision tree needed less time for data cleaning. Further, decision trees are not very complex unlike other such models, because they do not involve any complicated equations or formulae, thereby making it simpler to understand as well and make changes accordingly. Hence, the Decision Tree algorithm proved to be the most suitable model and has been applied in the proposed system.

The application developed in the system has been tested using the parking images and the following results were obtained. The user is provided with two options - to see all vacant parking slots nearby and also then nearest parking slot as per their location. Figure 4 represents all nearby parking slots. The user's location is represented as a label the Figure, and all nearby parking slots are represented as markers ' $P$ '. SimilarlyFigure5shows the user only the Nearest empty parking slot. It is clearly the nearest one to the user when compared to all other empty parking slots in Figure4. In Figure 6, the user is directed to the nearest parking slot by means of the route displayed in the figure. This route has been configured by implementing the Direction API. This API along with the travel time parameter takes into account other parameters like distance and number of turns in the route while calculating the route for the user. Thus, this idea of a smart parking system that has been brought out in this paper not only lets the user know about all vacant and nearest parking spots nearby the user, but also displays the route to the nearest parking spot, all of this provided in prior.

Published By:

Blue Eyes Intelligence Engineering

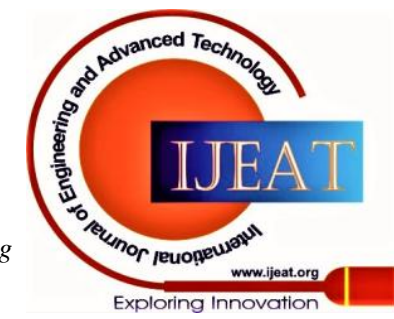




\section{Parking Assist using Convolution Neural Networks}

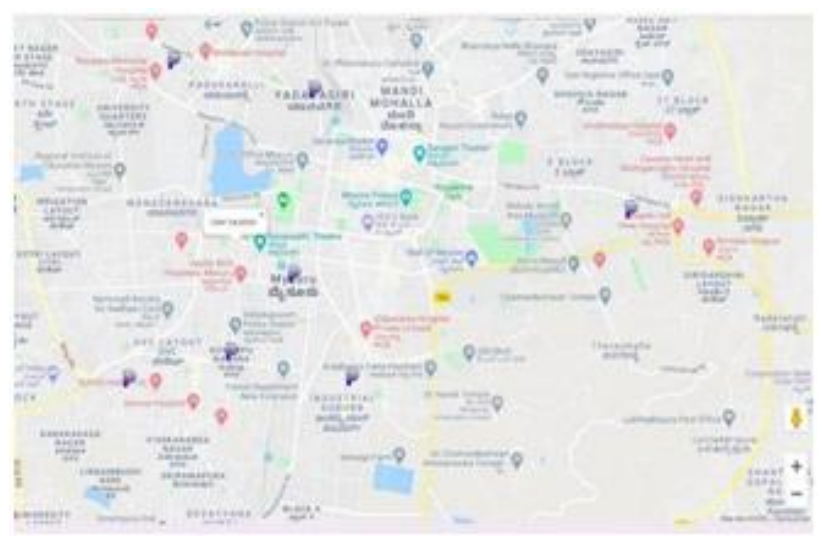

Figure 4 - Fetch all parking slots nearby page

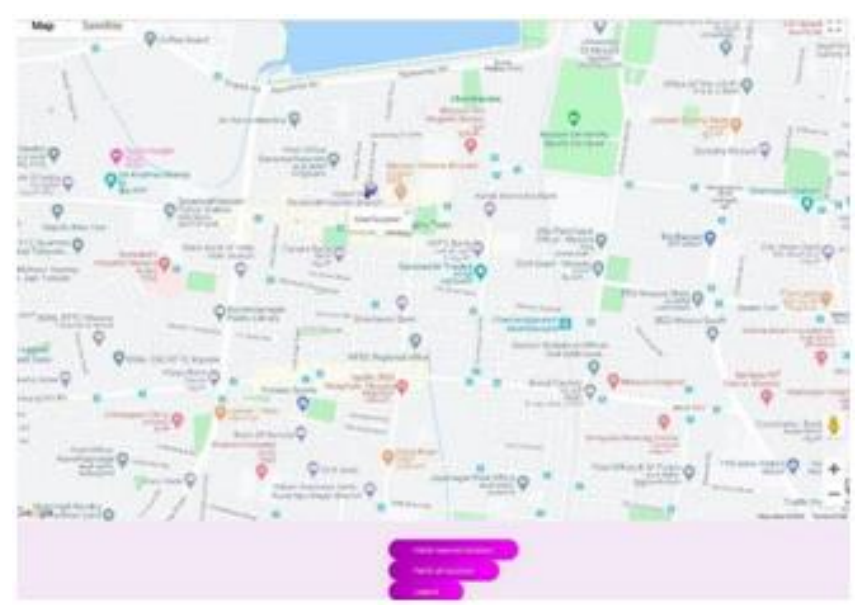

Figure 5 - Fetch Nearest parking slots nearby page

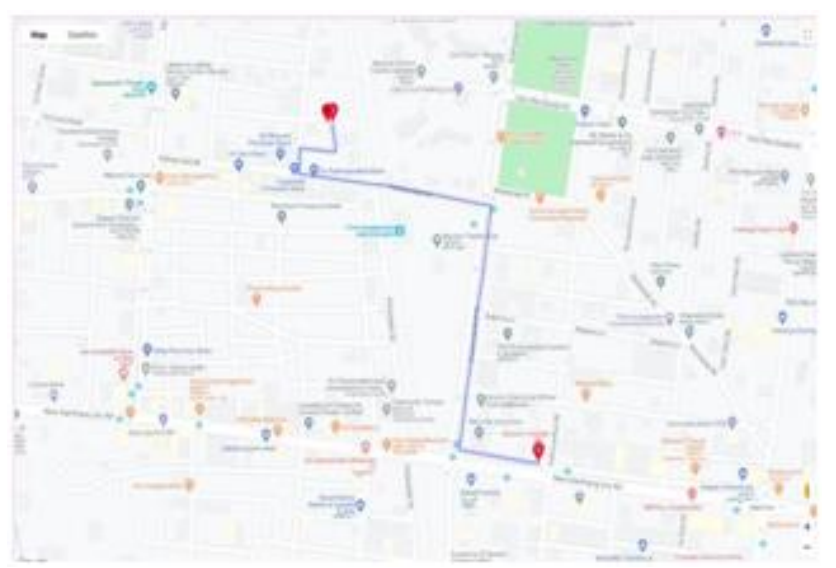

Figure 6 - Guide user to slot page

\section{CONCLUSION}

This paper presents a model of a Parking assist to drivers which would help the locate nearby empty parking slots using the technique of ResNe-50, a Convolution neural Network. This was developed with the intention of reducing the hassles of parking like wastage of time, fuel, pollution and also to make the process of parking a much easier and convenient task for drivers. The project is basically operated by the end user by means of a web app, which would provide the necessary information related to the nearby parking spaces and in order to obtain parking information, users need to permit the web app to fetch current location of user. The application shows clearly all the nearby vacant parking spaces from which the user can choose any of those empty slots. This way the user would not have to wait at a place looking for a parking spot, and instead can find it when he/she is on route. Thus, this paper has presented one such idea of a parking system which lets the user know about all vacant and nearest parking slot in prior and also guide user to the same. The problem simplified by this will also be reduced to a great extent and aid in helping people overcome the problems related to parking. This was the intent behind the project and has been achieved to the best possible extent.

\section{REFERENCES}

1. Valipour, Mennatullah Siam, Eleni Stroulia, Martin JagersandSepehr, "Parking Stall Vacancy Indicator System Based on Deep Convolutional Neural Networks", University ofAlberta,2016.

2. Vijay Paidi and Hasan FleyehDalarna "Parking Occupancy Detection using Thermal Camera" ,Dalarna University,School of technology and Business Studies,Borlange, Sweden.

3. Apeksha P Bari,Manali A Parab,Amey U Kokate and Nida Parkar, "IOT based smart parking system", IOSRJounal of Engineering,vol 15,pp28-31,2018

4. DebadityaAcharya,Weilin Yan and Kourosh Khoshellam,"Real-time image based parking Occupancy detection using deeplearning", Infrastructure Engineering, University of Melbourne.

5. J Cynthia, CBharathiPriya, C A Gopinath, "IoT based smart parking management system", International Journalof Recent Technology and Engineering, vol7,2018

6. Mr.BasavarajuSR,“Automatic Smart Parking System using Internet of Things (IOT)", RV College of engineering,vol 5,2015

7. Ms. MarziaAlam, “Automated Car Parking System”,B.S Thesis ,BRAC University, DhakaBangladesh.

8. Julien Nyambal, RichardKlein," Automated Parking Space Detection Using Convolutional Neural Networks", School of Computer Science and Applied Mathematics,University of the Witwatersr and Johannesburg.

9. M. Patil,V.N. Bhonge,"wireless Sensor Network and RFID for Smart Parking System", in IJETAE, Vol. 3, No. 4, 2013.

10. NazishFatima,PratikshaJagtap,AkshayaNatkar,SnehalC houdhary,"IoTbasedsmartparkingsystem martcities", International Journal ofAdvanced Research,Ideas and Innovations in Technology.

11. E Cassin Thangam,M Mohan, J Ganesh,C V Sukhesh,

a. / "Internet of Things(IoT) based Smart parking reservation system using Raspberry-pi”, International Journal of Applied Engineering Research vol 13, pp.5759-5765.

12. Dr RaghavenderRao, "Automatic Smart Parking system using Internet of Things (IoT)", International Conference on New Frontiers of Engineering, Science, Management and Humanities

\section{AUTHORS PROFILE}

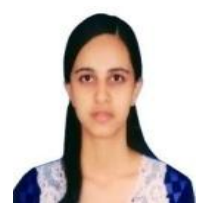

Swasthi.B.S, pursuing B.E in Computer Science and Engineering in Vidyavardhaka College of Engineering, Mysuru, currently in $8^{\text {th }}$ semester. Interested in research in the domain of Machine learning, Human- compute Interaction and Artificial Intelligence.

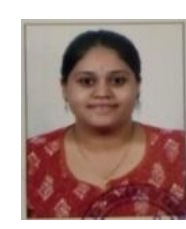

Anagha.R, pursuing B.E in Computer Science and Engineering in Vidyavardhaka College of Engineering, Mysuru, Currently in $8^{\text {th }}$ semester. Interested in the domain of Machine Learning and Internet of things.

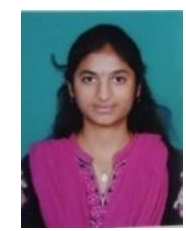

Arpitha.S, pursuing B.E in Computer Science and Engineering in Vidyavardhaka College of Engineering, Mysuru, Currently in $8^{\text {th }}$ semester. Interested in the domain of Big Data and Internet of things.

Published By:

Blue Eyes Intelligence Engineering

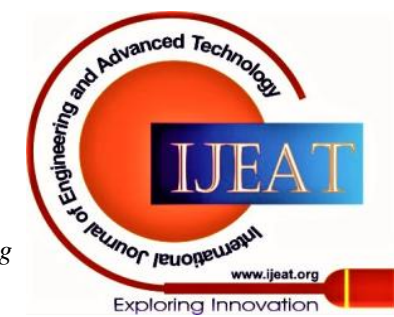


Sanjay.B.S, pursuing B.E in Computer Science and Engineering in Vidyavardhaka College of Engineering, Mysuru, Currently in $8^{\text {th }}$ semester. Interested in research in the domain of Machine learning, Internet of things and Artificial intelligence.

Harshitha K, Assistant Professor, Computer Science and Engineering, Vidyavardhaka College of Engineering, Mysuru. Completed B.E. and M.tech from Visvesvaraya technological University. Interested in research in the domain of Data Analytics and IoT. Published papers in conferences.

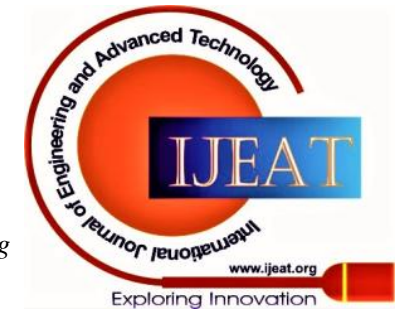

